\title{
Nanostructured metal oxide-based biosensors
}

\author{
Pratima R. Solanki, Ajeet Kaushik, Ved V. Agrawal and Bansi D. Malhotra* \\ National Physical Laboratory, India
}

Nanostructured metal oxides (NMOs) have recently become important as materials that provide an effective surface for biomolecule immobilization with desired orientation, better conformation and high biological activity resulting in enhanced sensing characteristics. Nanostructured metal oxides with unique optical, electrical and molecular properties along with desired functionalities and surface charge properties provide interesting platforms for interfacing biorecognition elements with transducers for signal amplification. In this review, we discuss the various approaches that have been adopted for improving the performance of NMO-based biosensors for clinical and non-clinical applications. The performance of an NMO-based biosensor can be improved by tailoring the properties of the metal oxide-biomolecule interface through engineering of morphology, particle size, effective surface area, functionality, adsorption capability and electron-transfer properties. These interesting NMOs are expected to find applications in a new generation of miniaturized, smart biosensing devices.

\begin{abstract}
A mong the various types of nanomaterials that have been developed, nanostructured metal oxides (NMOs) have recently aroused much interest as immobilizing matrices for biosensor development (Figure 1) [1-9]. Nanostructured oxides of metals such as zinc, iron, cerium, tin, zirconium, titanium, metal and magnesium have been found to exhibit interesting nanomorphological, functional biocompatible, non-toxic and catalytic properties. These materials also exhibit enhanced electron-transfer kinetics and strong adsorption capability, providing suitable microenvironments for the immobilization of biomolecules and resulting in enhanced electron transfer and improved biosensing characteristics. Various morphologies of NMOs have been obtained using a variety of methods, including soft templating for the preparation of nanorods and nanofibers [10], sol-gel methods for the production of three-dimensional (3D) ordered rough nanostructures [6,11], radiofrequency sputtering for rough nanostructures [4] and hydrothermal deposition for nanoparticles with controlled shape [12]. All of these NMOs have been reported to have potential applications in biosensors. Recently, the optical, electrical and magnetic properties of NMOs have been reported to be enhanced through the incorporation of nanoparticles of conducting or semiconducting materials such as carbon nanotubes, graphene, gold and silver, as well as quantum dots of various semiconductors, with advantages for improved biosensor characteristics [13-16]. It is expected that the judicious application of an NMO may lead to the fabrication of novel biosensing devices with enhanced signal amplification and coding strategies for bioaffinity assays and efficient electrical communication with redox biomolecules/ enzymes that may address future diagnostic needs.

The unique properties of NMOs offer excellent prospects for interfacing biological recognition events with electronic signal transduction and for designing a new generation of bioelectronics devices that may exhibit novel functions. The controlled preparation of an $\mathrm{NMO}$ is considered to play a significant role in the development of biosensors. Efforts are being made to explore the prospects and future challenges of NMOs for the development of biosensing devices and the evolution of new strategies for bioaffinity assays and efficient electrical
\end{abstract}

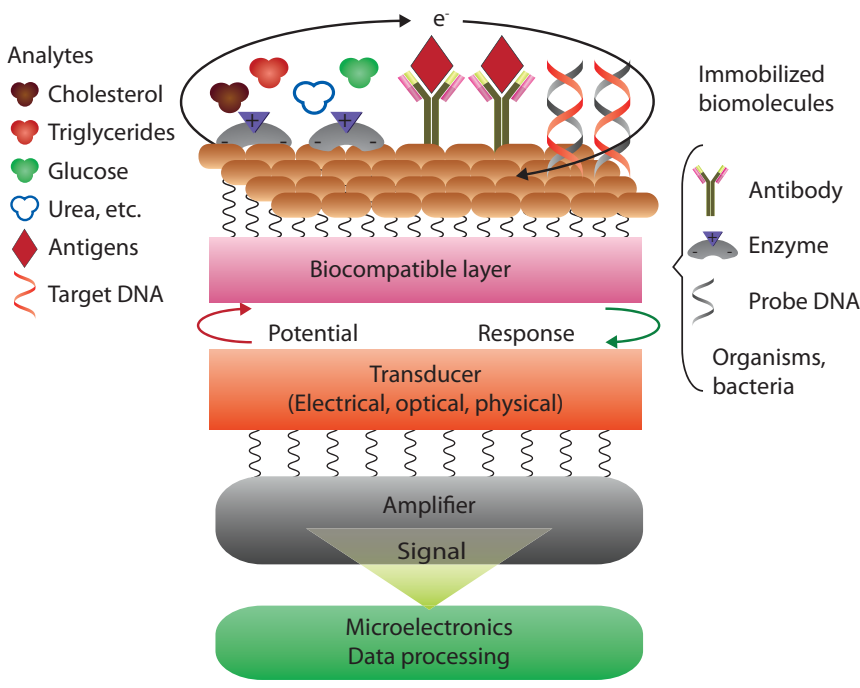

Figure 1. Schematic structure and operating principle of a biosensor

communication. In this review, we focus on developments over the past five years in NMO-based biosensors for clinical and non-clinical applications (Figure 2).

\section{Fundamentals of nanostructured metal oxides for biosensing}

Among the various immobilizing matrices that have been developed, NMOs have exceptional optical and electrical properties due to electron and phonon confinement, high surface-to-volume ratios, modified surface work function, high surface reaction activity, high catalytic efficiency and strong adsorption ability. For these reasons, NMOs have been utilized to increase the loading of biomolecules per 


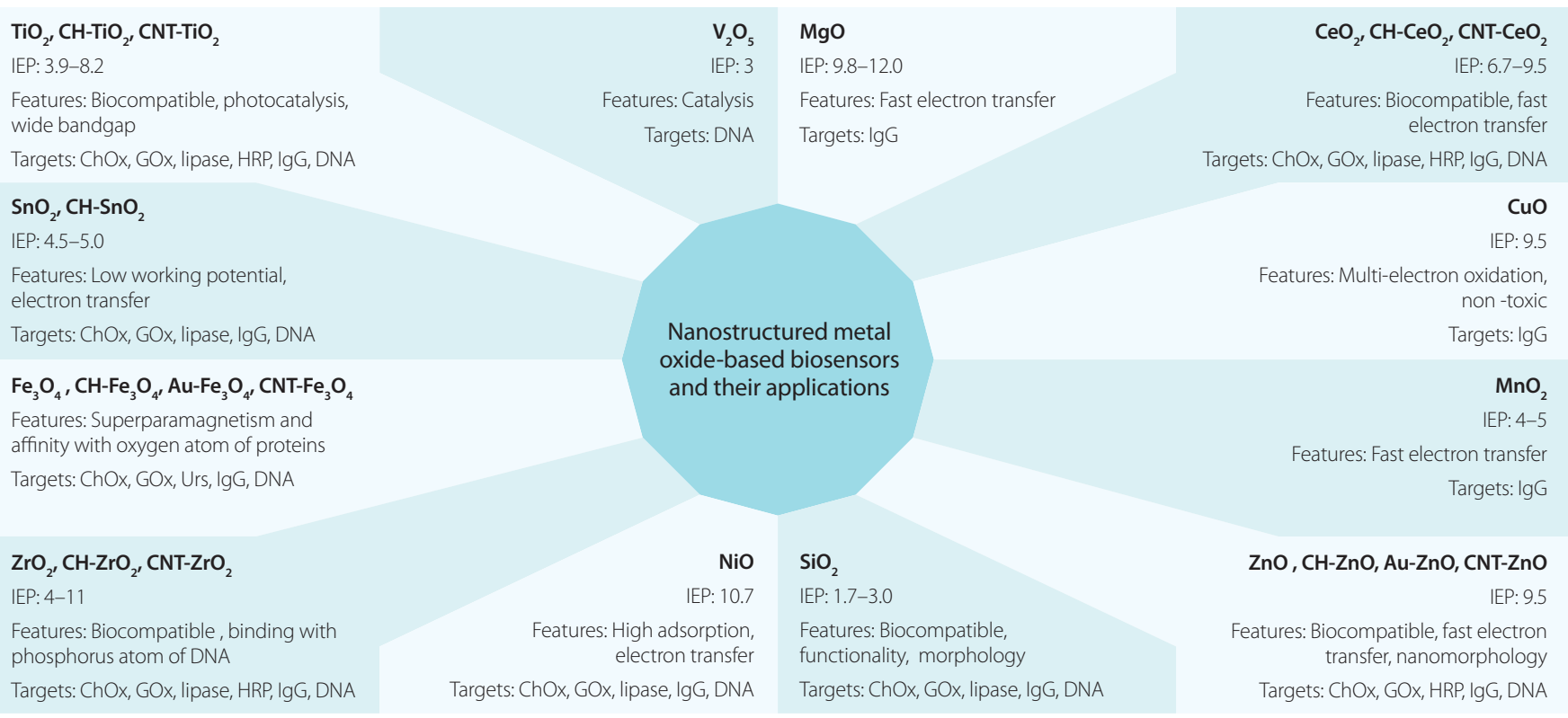

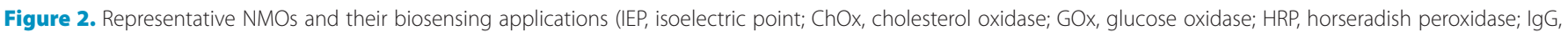
immunoglobulin G; Urs, urease)

unit mass of particles. The use of an NMO allows for the possibility of many new signal transduction technologies in biosensors arising from the sub-micrometer dimensions that can be utilized for simple and rapid in vivo analysis [17].

A biosensor is an integrated miniaturized device that employs a biological element (antibody, enzyme, receptor protein, nucleic acid, whole cell or tissue section) as a sensing element coupled to a transducer for signal detection. A biosensor utilizes the selectivity of the biomolecule and the processing power of modern microelectronics and optoelectronics and is hence a powerful analytical tool with applications in medical diagnostics and other areas. Due to their specificity, portability, rapid response time and low cost, biosensors are projected to play a critical role in both clinical and non-clinical applications. The prevailing miniaturization tools allow packing of numerous microscopic electrodes along with transducers into a small footprint of a biochip device, leading to the design of high-density bioarrays [18].

Investigations relating to the effect of the optical and electrochemical properties of NMOs, suspended media, solid-liquid interfaces and nanobiointerfaces are being conducted for biosensor applications. To fabricate an efficient biosensor, it is crucial to select an NMO that is suitable for immobilization of the desired biomolecule (Figure 3). The interface formed due to binding between an NMO and a biomolecule is known to affect the performance of a biosensor. The formation and properties of a nanobiointerface depend on the nature of the NMO; parameters like effective surface area, surface charge, energy, roughness and porosity, valence/conductance states, functional groups, physical states and hygroscopic nature all affect the formation of a biointerface. In general, biomolecules bind with metal oxide nanoparticles via physical adsorption or chemical binding. The physical adsorption of a biomolecule arising due to weak interactions (e.g. van der Waals, electrostatic, physisorption) depends on the surface morphology, reaction medium and net surface charge. Short-range forces arising via charge, steric, depletion and solvent interactions also play an important role in the preparation of a nanobiointerface. Covalent binding of a biomolecule to an NMO depends on the availability of functional groups, which can be prepared by appropriate engineering of chemical reactions. An effective NMO biointerface can help a biomolecule retain its biological activity with high stability by establishing a biocompatible microenvironment. The rate of electron transfer depends on the characteristics of the NMO-biomolecule interactions [19]. There is thus considerable opportunity for the development of biosensors with improved sensitivity and detection limits, as well as lower cost and extended shelf life, through the use of NMOs. Various biomolecules, including enzymes, nucleic acids and antibodies, can be immobilized on NMOs (Figure 3), and some of the most promising biomolecular systems are reviewed below.

\section{Enzyme-immobilized NMO-based biosensors}

An enzyme biosensor is based on a biochemical reaction that occurs between an analyte and a biocatalyst immobilized on a suitable substrate. Nanostructured metal oxides have excellent prospects for interfacing biological recognition events with electronic signal transduction as a new generation of biosensors in which active enzymes sites are coupled directly with an NMO electrode resulting in direct electron transfer between the enzyme and NMO with improved biosensing properties [17].

Nanostructured metal oxide-based electrodes provide a biocompatible electroactive surface for enzyme immobilization with improved conformation, orientation and biological activity [17]. An important challenge in the development of amperometric bioelectrodes is the establishment of satisfactory electrical communication between the

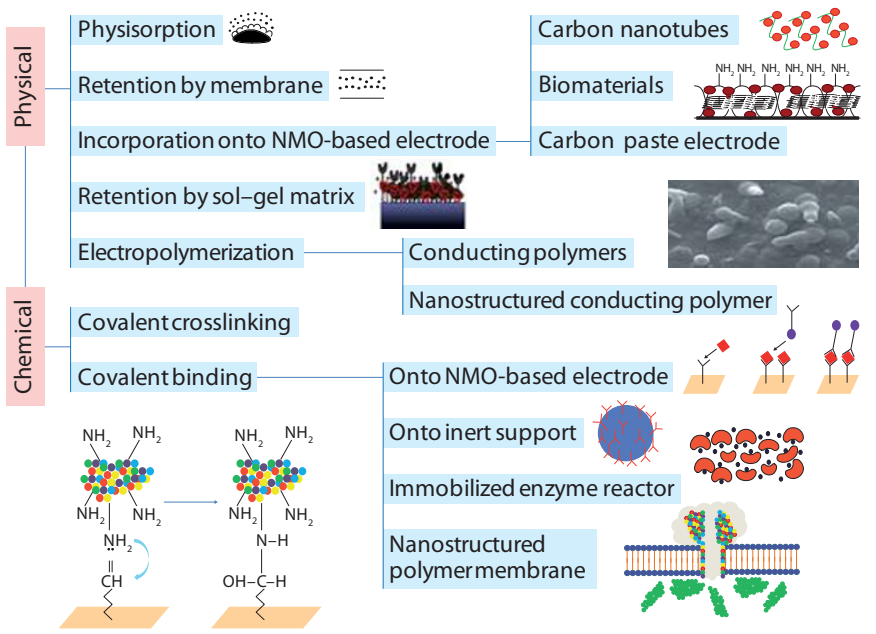

Figure 3. Various immobilization schemes for biosensor fabrication 
active sites of biomolecules and the NMO electrode surface. For example, the redox centers of most oxidoreductases are electrically insulated by protein shells. As a result, enzymes cannot be oxidized or reduced at the electrode surface at any potential. The possibility of direct electron transfer between enzymes and the NMO-based electrode surface could pave the way for the development of superior reagentless biosensing devices, as it may obviate the need for co-substrates or mediators, allowing efficient transduction of biorecognition events. The close approach of a redox site to an electrode is often impossible without the application of a large overpotential that could lead to poisoning of the electrode surface and the denaturation of enzymes. Advances in achieving direct electron transfer can be made by modification of an enzyme or electrode surface with a mediator or a nanoparticle.

Many approaches have been followed for the engineering of biosensor components, particularly in the area of NMO-based immobilizing matrices and transducers (electrochemical and optical), to obtain enhanced biosensing performance in various applications. Nanostructured metal oxides with useful optical, electrochemical and molecular properties have been prepared by various syntheses routes, as well as through the doping of nanomaterials and fabrication of organic/inorganic nanocomposites. In particular, NMOs with engineered morphology, size (for biocompatibility), functionality, adsorption capability and high biomolecule loading capacity have been found to provide enhanced electron transport between biomolecules and electrodes. The microenvironment provided by an NMO helps an enzyme to retain its conformation with maximum biological activity, resulting in enhanced signal transduction and biosensor stability. The performance of a biosensor can also be affected by the choice of transducer (Figure 4). Sensitive and selective biosensors have been fabricated using the effect of surface plasmon resonance. However, such systems are complex and require considerable time, expense and expertise to develop. Electrochemical techniques such as cyclic voltammetry, on the other hand, are simple and can be utilized for the rapid and cost-effective development of biosensors. The characteristics of an electrochemical biosensor can be improved through the use of differential pulse voltammetry (DPV). Electrochemical impedance spectroscopy (EIS) is a non-destructive electrochemical analytical technique that has recently been used for signal transduction and which has been particularly useful for clarifying the mechanisms of charge transfer resistance arising due to electron transport between a biomolecule and the electrode surface. Electrochemical devices have advantages in terms of size, cost, detection limit, response time, long-term stability and power requirements, and therefore have great promise for a wide range of biomedical and environmental applications. Nanostructured metal oxides with various surface architectures have been used to produce a number of electrochemical biosensing devices with improved sensitivity and selectivity.

\section{Glucose oxidase}

There have been many attempts to immobilize glucose oxidase (GOx) on NMOs for the fabrication of a glucose biosensor. Metal oxide-based nanomaterials with high isoelectric point (IEP) have been found to be suitable for the immobilization of biomolecules with low IEP via electrostatic interactions. Improving the sensitivity and linearity of glucose biosensors using other NMOs and their composites with desired biopolymers has been the focus of many studies in this area.

A single-crystal $\mathrm{NMO}$ consisting of $\mathrm{ZnO}$ nanocombs prepared in bulk by vapor-phase deposition provides a $3 \mathrm{D}$ porous network with high specific surface area and good biocompatibility for GOx, allowing GOx to be loaded at high densities [20]. This favorable NMO-based microenvironment offers high sensitivity $\left(15.33 \mu \mathrm{AmM}^{-1} \mathrm{~cm}^{-2}\right)$ and high affinity of GOx to glucose, achieving a Michaelis-Menten constant $\left(K_{\mathrm{m}}\right)$ of $2.19 \mathrm{mM}$. An NMO-based glucose biosensor based on $\mathrm{ZnO}$ nanotube arrays synthesized by chemical etching of $\mathrm{ZnO}$ nanorods and electrochemical deposition onto a gold surface to immobilize GOx also displays improved characteristics [21]. The

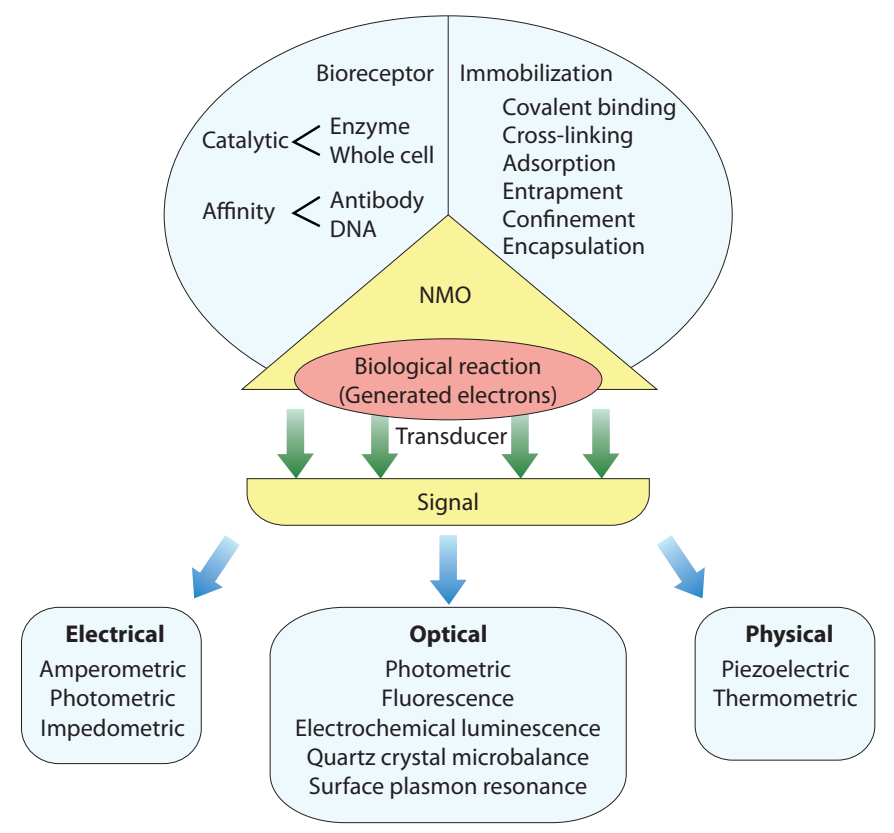

Figure 4. Various types of transducers for biosensing

intrinsic nanotubular structure of $\mathrm{ZnO}$ provides good response without a mediator, wide linear range, low detection limit $(1 \mu \mathrm{M})$, sensitivity of $21.7 \mu \mathrm{AmM}^{-1} \mathrm{~cm}^{-2}$ and $K_{\mathrm{m}}$ of $21.7 \mathrm{mM}$.

A highly sensitive amperometric biosensor for continuous glucose monitoring based on a single $\mathrm{ZnO}$ nanofiber (195-350 nm in diameter) synthesized by electrospinning has been shown to have high and reproducible sensitivity of $70.2 \mu \mathrm{AmM}^{-1} \mathrm{~cm}^{-2}$ due to its high specific surface area and higher capacity for enzyme loading, providing a microenvironment that helps GOx retain its bioactivity and enhanced thermal stability over a temperature range of $20-85^{\circ} \mathrm{C}$ [22]. A recently fabricated mediator-free glucose biosensor based on GOx and horseradish peroxidase (HRP) immobilized on a carbon-decorated $\mathrm{ZnO}(\mathrm{C}-\mathrm{ZnO})$ nanowire array electrode provides high sensitivity and a low detection limit for glucose [23]. The $\mathrm{C}-\mathrm{ZnO}$ nanowire array is a fast, direct electrochemical platform for GOx. Glucose oxidase has also been adsorbed onto hexagonal $\mathrm{ZnO}$ nanorods on the tip of a silver-coated borosilicate glass capillary $(0.7 \mu \mathrm{m}$ in diameter) for intracellular glucose measurement in human adipocytes and frog oocytes [24]. $\mathrm{ZnO}$ nanowires have been found to be biosafe with high ionic bonding (60\%), and to be insoluble at biological $\mathrm{pH}$. This intracellular biosensor exhibits fast response times and wide linear range.

Nanostructured $\mathrm{CeO}_{2}$, with a high IEP of 9.4, exhibits a high electron-transfer rate constant and good surface coverage, allowing high enzyme loading and fast electron transfer between the active GOx site and the electrode. Saha et al. [25] deposited nanoporous thin $\mathrm{CeO}_{2}$ films onto platinum-coated glass plates using pulsed laser deposition for the immobilization of GOx, producing a glucose biosensor with a linear response to glucose and a $K_{\mathrm{m}}$ value as low as $1.01 \mathrm{mM}$. However, this glucose biosensor is expensive to produce, requires considerable expertise to realize, and has only a limited glucose detection range and poor detection limit. A simple sol-gel method can instead be used to produce a nanostructured $\mathrm{CeO}_{2}$ film for the fabrication of a glucose biosensor [6].

Wen et al. [26] used a novel approach to fabricate a biofunctional nanocatalyst comprising evenly dispersed $\mathrm{Au} / \mathrm{Pt}$ hybrid nanoparticles assembled onto $\mathrm{NH}_{2}$-functionalized $\mathrm{TiO}_{2}$ colloid spheres via electrostatic interactions. The nanoparticulate $\mathrm{TiO}_{2}-\mathrm{Au} / \mathrm{Pt}$ has been reported to be an excellent performance-enhancing material, providing superior catalytic reduction of $\mathrm{H}_{2} \mathrm{O}_{2}$. $\mathrm{NH}_{2}$-functionalized nanoporous $\mathrm{TiO}_{2}$ assembled with platinum nanoparticles has also been utilized for glucose biosensing 


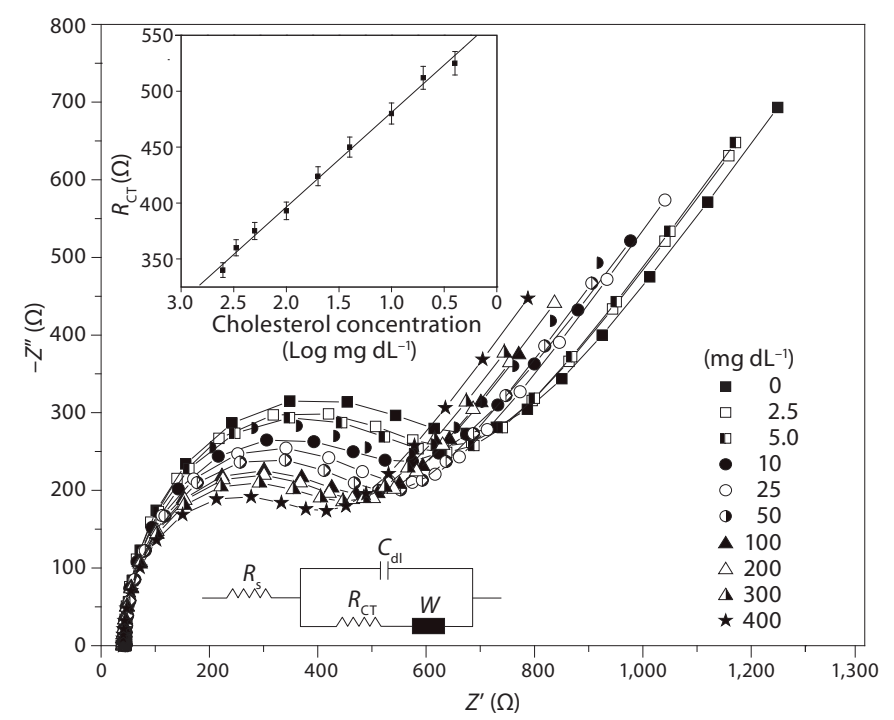

Figure 5. Electrochemical impedance spectroscopy response of a ChOx/nano$\mathrm{Fe}_{3} \mathrm{O}_{4} /$ ITO bioelectrode as a function of cholesterol concentration at bias potential of $0.06 \mathrm{~V}$. Inset shows a calibration curve between charge-transfer resistance $\left(R_{\mathrm{CT}}\right)$ value and the logarithm of cholesterol concentration.

via the immobilization of $\mathrm{GOx}$ [27]. Such $\mathrm{CH} / \mathrm{TiO}_{2}-\mathrm{Pt}$ nanoparticle films provide a low detection limit $(0.25 \mu \mathrm{M})$ due to enhanced electron transfer between GOx and the electrode surface. The results of these studies have implications regarding the development of other enzyme electrodes based on these NMOs.

A nanocomposite film consisting of GOx immobilized on $\mathrm{Fe}_{3} \mathrm{O}_{4}$ nanoparticles $(-22 \mathrm{~nm}$ in diameter $)$ well-dispersed in $\mathrm{CH}$ for mediatorfree glucose detection has been shown to provide enhanced electron transfer resulting in improved sensing characteristics including better linearity, higher sensitivity and lower $K_{\mathrm{m}}(0.141 \mathrm{mM})$ [28]. The $\mathrm{Fe}_{3} \mathrm{O}_{4}$ nanoparticles in this nanocomposite system provide a high electroactive surface area that contributes to the oriented immobilization of GOx. $\mathrm{MgO}$-based polyhedral nanocages and nanocrystals prepared by simple non-catalytic thermal evaporation have been utilized for the fabrication of glucose biosensors with high and reproducible sensitivity of 31.6 $\mu \mathrm{A} \mu \mathrm{M}^{-1} \mathrm{~cm}^{-2}$ with a response time of less than $5 \mathrm{~s}$, a linear dynamic range of 1.0-9.0 $\mu \mathrm{M}$ and a detection limit as low as $68.3 \pm 0.02 \mathrm{nM}$ [29]. A mediator-free glucose biosensor based on the electrochemical codeposition of nanoporous $\mathrm{NiO}_{x}$ nanoparticles and GOx onto a glassy carbon electrode (GCE) has been reported [30]. The surface coverage and heterogeneous electron transfer rate constant $\left(k_{\mathrm{s}}\right)$ of GOx immobilized on $\mathrm{NiOx}$ film are $0.945 \mathrm{pmolcm}^{-2}$ and $25.2 \pm 0.5 \mathrm{~s}^{-1}$, indicating that the $\mathrm{NiO}$ nanoparticles have high enzyme loading ability and fast electron transfer between GOx via conducting tunnels. Such a sensor could be useful for real-time monitoring of glucose and the research opens a new direction for the utilization of $\mathrm{NiO}$ and $\mathrm{MgO}$ nanostructures as an electron mediator for efficient biosensors.

\section{Cholesterol oxidase}

Many NMOs have been used to fabricate cholesterol biosensors using cholesterol oxidase (ChOx). A nanostructured $\mathrm{ZnO}$ film with a wide bandgap $(3.37 \mathrm{eV})$ and large excitation binding energy $(60 \mathrm{eV})$, prepared using sol-gel process, has been used for the immobilization of ChOx (IEP 4.7) to fabricate a cholesterol biosensor with a wide linear range $\left(5.0-400 \mathrm{mg} \mathrm{dL}^{-1}\right)$, low $K_{\mathrm{m}}\left(0.98 \mathrm{mg} \mathrm{dL}^{-1}\right)$, and high sensitivity (59 $\mathrm{nA} \mathrm{mg}^{-1} \mathrm{dL} \mathrm{cm}^{-2}$ ) [31]. A thin porous $\mathrm{ZnO}$ film obtained by radiofrequency magnetron sputtering under high pressure $(50 \mathrm{~m}$ Torr $)$ has been utilized to fabricate a cholesterol biosensor with a linear range from 0.65 to $10.34 \mathrm{mM}$ and $K_{\mathrm{m}}$ values as low as $2.1 \mathrm{mM}$ [4]. These cholesterol biosensors could be used to estimate cholesterol concentration in serum/blood samples.
A highly sensitive amperometric cholesterol biosensor based on platinum-incorporated fullerene-like $\mathrm{ZnO}$ hybrid nanospheres (50-200 nm) has been fabricated by electrodeposition of the nanospheres onto a GCE [32]. This biosensor exhibits improved sensitivity of $1.886 \mathrm{~mA}$ $\mathrm{mM}^{-1} \mathrm{~cm}^{-2}$ resulting in increased enzyme activity. It has been found that the combination of $\mathrm{ZnO}$ and platinum nanoparticles facilitates lowpotential $(0.2 \mathrm{~V})$ amperometric detection of cholesterol with enhanced anti-interference ability. Improved sensitivity and a low detection limit have been achieved for a cholesterol biosensor prepared using a modified $\mathrm{ZnO}$ nanostructure with a gold electrode [33]. This bioelectrode shows high and reproducible sensitivity of $23.7 \mu \mathrm{AmM}^{-1} \mathrm{~cm}^{-2}$, detection limit of $0.37 \pm 0.02 \mathrm{nM}$ and linear range of $1.0-500 \mathrm{nM}$. However, the observed higher value of $K_{\mathrm{m}}(4.7 \mathrm{mM})$ indicates poor interaction between $\mathrm{ZnO}$ and $\mathrm{ChOx}$.

A cholesterol biosensor based on a nanocomposite film consisting of $\mathrm{SnO}_{2}$ nanoparticles and $\mathrm{CH}$ has been reported by Ansari et al. [34]. The high sensitivity of this sensor has been attributed to the increased adsorption ability, high electrocatalytic activity and good biocompatibility of the $\mathrm{CH}-\mathrm{SnO}_{2} / \mathrm{ITO}$ nanocomposite for ChOx. It appears that the hydrogen bonds in this nanocomposite facilitate maintenance of the native configuration of ChOx. The lower $K_{\mathrm{m}}(3.8 \mathrm{mM})$ obtained for this nanocomposite is due to enhanced affinity of $\mathrm{ChOx}$ towards cholesterol, indicating strong binding of $\mathrm{ChOx}$ to the $\mathrm{CH}-\mathrm{SnO}_{2}$ surface. Electrodeposited $\mathrm{CoO}_{x}$ nanoparticles have been used both for entrapment of $\mathrm{ChOx}$ and as a mediator for faster oxidation [35]. The porous surface of $\mathrm{CoO}_{x}$ acts as a transport channel for the substrate, reducing the mass transfer resistance and enhancing the efficiency of biocatalysis. This sensor shows linearity up to $50 \mathrm{mM}$, sensitivity of $43.5 \mathrm{nAmM}^{-1} \mathrm{~cm}^{-2}$ and a detection limit of $4.2 \mathrm{mM}$. The apparent $K_{\mathrm{m}}$ and the response time of this biosensor are $0.49 \mathrm{mM}$ and $15 \mathrm{~s}$.

A nanostructured $\mathrm{Fe}_{3} \mathrm{O}_{4}$-based EIS platform has been utilized for cholesterol detection through the immobilization of $\mathrm{ChOx}$ [36]. In this system, the charge-transfer resistance decreases with increasing cholesterol concentration (Figure 5). This biosensor exhibits improved linearity, a low detection limit and low $K_{\mathrm{m}}\left(0.8 \mathrm{mg} \mathrm{dL}^{-1}\right)$. In spite of these interesting developments, there remains considerable room for improvement of NMO-based cholesterol biosensors. For example, no NMO-based biosensor has been developed for the estimation of total cholesterol concentrations.

\section{Horseradish peroxidase}

A waxberry-like $\mathrm{ZnO}$ microstructure with $8-10 \mathrm{~nm}$-diameter nanorods on a GCE has been used for the immobilization of HRP [37]. This matrix has been found to be an excellent material for the immobilization of HRP, providing rapid electron transfer and allowing the fabrication of efficient biosensors with high electrical conductivity, large surface area, abundant oxygen vacancies and good biocompatibility. A flower-like $\mathrm{ZnO}$-gold nanoparticle-Nafion nanocomposite bearing immobilized HRP has been reported for $\mathrm{H}_{2} \mathrm{O}_{2}$ detection via direct electron transfer [38]. This sensor has a linear response over a wide range of $\mathrm{H}_{2} \mathrm{O}_{2}$ concentration $(15-1,100 \mu \mathrm{M})$ and a detection limit of $9.0 \mu \mathrm{M}$, indicating that this matrix provides a favorable microenvironment for the enzyme to retain its activity.

$\mathrm{ZnO}$ nanorods have also been fabricated on gold wires by a hydrothermal reaction without a surfactant [39]. In this system, the ends of the gold wires were coated with a thin layer of $\mathrm{Zn}$-Au alloy to improve the nucleation and growth of $\mathrm{ZnO}$ nanostructures. This approach improved the performance of the biosensor by allowing the alternate immobilization of poly(sodium-4-styrenesulfonate) and HRP onto $\mathrm{ZnO}$ for $\mathrm{H}_{2} \mathrm{O}_{2}$ detection without the need for a mediator. This multilayered HRP sensor exhibits a wide linear range and low detection limit, and its sensitivity increases with the thickness of the immobilized HRP layer from $36.28 \mu \mathrm{A} \mathrm{mM}^{-1} \mathrm{~cm}^{-2}$ for a monolayer. Using a physisorption technique, HRP has been immobilized on nanostructured $\mathrm{CeO}_{2}$ [40]. The bioelectrode thus prepared exhibits low $K_{\mathrm{m}}(2.21 \mu \mathrm{M})$ and linearity for $\mathrm{H}_{2} \mathrm{O}_{2}$ detection over the range $1.0-170 \mu \mathrm{M}$. 
Various morphologies of nanostructured $\mathrm{MgO}$, including flowerlike nanostructures and nanotubes, have recently been reported. For example, a composite of $\mathrm{MgO}$ nanoparticles and $\mathrm{CH}$ has been developed for the immobilization of HRP for $\mathrm{H}_{2} \mathrm{O}_{2}$ detection in the presence of hydroquinone as a mediator [41]. This biosensor has a rapid response to $\mathrm{H}_{2} \mathrm{O}_{2}$ due to the excellent electrocatalytic behavior of $\mathrm{MgO}$. A linear relationship is obtained in the concentration range of $0.1-1,300 \mathrm{mM}$ with a detection limit of $50 \mu \mathrm{M}$. However, this sensor can be utilized for only a few weeks before degrading.

An $\mathrm{SnO}_{2}$ nanorod array-based biosensor produced by Liu et al. [42] has high sensitivity $\left(0.379 \mathrm{mAmM}^{-1} \mathrm{~cm}^{-2}\right)$, a low detection limit $(0.2 \mu \mathrm{M})$, high selectivity and low $K_{\mathrm{m}}(33.9 \mu \mathrm{M})$. These single-crystalline $\mathrm{SnO}_{2}$ nanorods were grown on an alloy substrate and provide a large number of void spaces for HRP immobilization.

\section{Urease and glutamate dehydrogenase}

Solanki et al. [43] have co-immobilized urease (Urs) and glutamate dehydrogenase $(\mathrm{GLDH})$ on a nanocomposite consisting of nanostructured $\mathrm{ZnO}$ for application as a urea biosensor. This bioelectrode shows high sensitivity and a detection limit of $13.5 \mathrm{mg} \mathrm{dL}^{-1}$ with $K_{\mathrm{m}}$ of $6.1 \mathrm{mg} \mathrm{dL}^{-1}$, indicating good affinity of Urs-GLDH for urea. A nanocomposite of $\mathrm{ZnO}$ and $\mathrm{CH}$ [44] has been shown to provide improved biosensing characteristics due to its high electroactive surface area and the combined effect of $\mathrm{CH}$ and $\mathrm{ZnO}$ nanoparticles resulting in increased loading of Urs-GLDH and improved charge transport. This biosensor exhibits high linearity and low $K_{\mathrm{m}}\left(4.92 \mathrm{mg} \mathrm{dL}^{-1}\right)$ due to enhanced affinity of enzymes toward the nanobiocomposite. The affinities of Urs and GLDH are also high for a nanobiocomposite film of superparamagnetic $\mathrm{Fe}_{3} \mathrm{O}_{4}$ nanoparticles and $\mathrm{CH}$ due to a low $K_{\mathrm{m}}$ of $0.56 \mathrm{mM}$ [45]. The $\mathrm{Fe}_{3} \mathrm{O}_{4}$ nanoparticles increase the active surface area for the immobilization of enzymes, enhanced electron transfer and increased the shelf-life of the electrode. It will be interesting to utilize these bioelectrodes for estimation of urea concentrations in clinical samples.

\section{Lipase}

Lipase immobilized on magnetic nickel-ferrite nanoparticles at the gate of an ion-selective field-effect transistor has been used to detect tributyrin, trioctanoate and triolein concentrations in the range of $0.1-1.5 \%$ [46]. Nanostructured $\mathrm{CeO}_{2}$ films ( $-35 \mathrm{~nm}$ thick) have also been used for the fabrication of a lipase-based tributyrin biosensor. This biosensor exhibits good linearity, low detection limit, long shelf-life and low $K_{\mathrm{m}}\left(22.27 \mathrm{mg} \mathrm{dL}^{-1}\right)$. A lipase-based biolectrode of nanostructured $\mathrm{CeO}_{2}$ and indium tin oxide (ITO) prepared by Solanki et al. [47] similarly shows high affinity for tributyrin [47]. Efforts should be made to utilize these electrodes in NMO-based biosensing devices for estimation of triglyceride concentrations in blood.

\section{Other enzymes}

$\mathrm{MnO}_{2}$ nanoparticles with various structures, including amorphous $\mathrm{MnO}_{2}, \alpha-\mathrm{MnO}_{2}$ nanoparticles and $\beta-\mathrm{MnO}_{2}$ nanowires, electrochemically deposited on a GCE with $\mathrm{CH}$ hydrogel and choline oxidase have been used for the detection of choline [48]. Biosensors based on crystalline $\mathrm{MnO}_{2}$ respond to choline far more quickly than those based on amorphous $\mathrm{MnO}_{2}$ under amperometric measurement conditions. $\beta-\mathrm{MnO}_{2}$-based biosensors show a particularly fast response time. It has been found that the specific surface area, the amount of $\mathrm{MnO}_{2}$ trapped on the electrodes, the crystalline structure and even dimensionality play critical roles in determining the biosensing behavior. Such biosensors exhibit a linear range of $2.0-580 \mu \mathrm{M}$ for $\alpha-\mathrm{MnO}_{2}$ nanoparticles and $1.0-790 \mu \mathrm{M}$ for $\beta-\mathrm{MnO}_{2}$ nanowires with detection limits of 1.0 and $0.3 \mu \mathrm{M}$, respectively [48]. Luo et al. [49] fabricated a highly conductive $\mathrm{TiO}_{2}$-nanoneedle film as a support for the immobilization of cytochrome c to facilitate electron transfer between redox enzymes and electrodes. In this system, they found inherent enzymatic activity towards $\mathrm{H}_{2} \mathrm{O}_{2}$ released from human liver cancer cells.

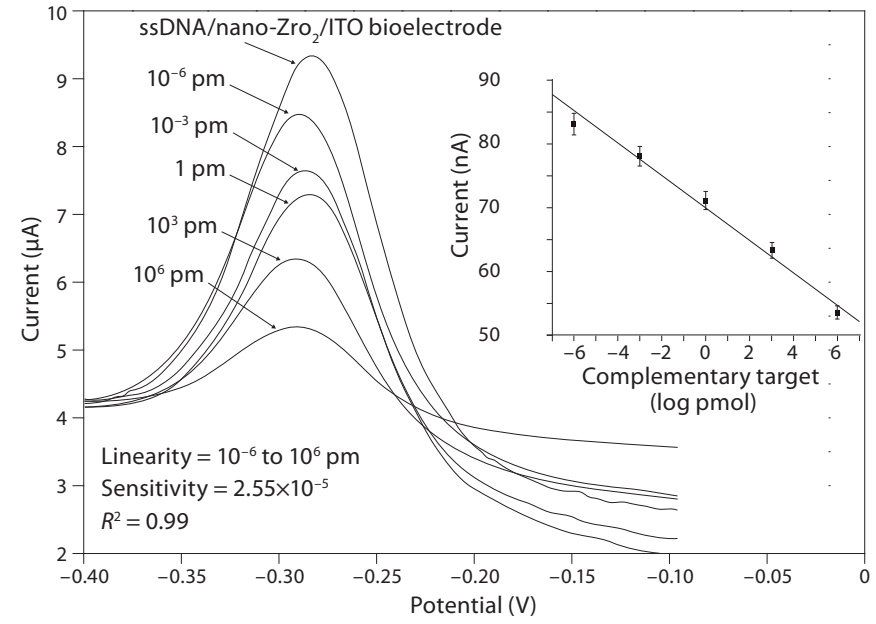

Figure 6. Differential pulse voltammetry response of a DNA/nano- $\mathrm{ZrO}_{2} / \mathrm{ITO}$ bioelectrode after hybridization with complementary target probe. Inset shows the calibration curve between current and the logarithm of target concentration.

\section{Nucleic acid-immobilized NMO-based biosensors}

Nanostructured metal oxide-based biosensors for DNA detection have recently aroused much interest due to recent revelations these could be used to convert a hybridization event into an analytical signal. Wang et al. [50] reported a DNA sensor based on $\mathrm{ZnO}$ nanowires, multiwalled carbon nanotubes (MWCNTs) and gold nanoparticles for nucleic acid detection. In their system, a single-stranded DNA (ssDNA) probe with a thiol group is covalently immobilized on the gold nanoparticle surface through Au-S bonding. This DNA biosensor can detect target DNA at concentrations of 0.10 to $10^{5} \mathrm{pM}$ with a detection limit of $0.35 \mathrm{pM}$ using $\left[\mathrm{Ru}\left(\mathrm{NH}_{3}\right)_{6}\right]^{3+}$ as an intercalator.

A DNA biosensor based on a composite of $\mathrm{ZnO}$ nanoparticles, MWCNTs and $\mathrm{CH}$ on a GCE has been used for hybridization detection by DPV using methylene blue as an indicator [51]. This sensor can discriminate among different DNA sequences related to the phosphinothricin acetyl transferase gene in transgenic corn, with a detection limit of $2.8 \mathrm{pM}$ of target sequence. Well-dispersed $\mathrm{Cu}_{2} \mathrm{O}$ hollow microspheres of $\mathrm{Cu}_{2} \mathrm{O}$ nanoparticles synthesized in aqueous solution at $25^{\circ} \mathrm{C}$ with polyvinylpyrrolidone as a surfactant have been used for hepatitis $\mathrm{B}$ virus detection [52]. Efforts should be made to improve the sensitivity of nucleic acid sensors.

Zuo et al. [53] fabricated a DNA biosensor by immobilizing ssDNA with a terminal 5'-phosphate group to a $\mathrm{ZrO}_{2}$ surface utilizing the strong affinity between the oxygen atoms of the phosphoric group and zirconium. This biosensor was found to have high sensitivity and selectivity for hybridization detection ( $\leq 20 \mathrm{nM}$ complementary DNA). A nanostructured $\mathrm{ZrO}_{2}$ film electrochemically deposited on gold has been used for the immobilization of 21-mer ssDNA specific to Mycobacterium tuberculosis, achieving a detection limit of $65 \mathrm{ng} \mathrm{mL}-1$ and a response time of less than $60 \mathrm{~s}$ [54]. An improvement in sensitivity and detection limit $(0.1 \mathrm{fM})$ for $M$. tuberculosis detection has been reported through the use of a nanocomposite film of 3-glycidoxypropyltrimethoxysilane and $\mathrm{Fe}_{3} \mathrm{O}_{4}$ nanoparticles for the covalent immobilization of 24-mer peptide nucleic acid [55]. This bioelectrode can be used for the detection of hybridization with the complementary sequence in sonicated M. tuberculosis genomic DNA within $90 \mathrm{~s}$.

A sol-gel-derived nanostructured $\mathrm{ZrO}_{2}$ film has similarly been used for the immobilization of 17-base ssDNA identified from the 16s rRNA coding region of Escherichia coli [56]. This bioelectrode exhibits a high selectivity and sensitivity for hybridization, with linearity in the range of $10^{-6}$ to $10^{6} \mathrm{pM}$ of complementary DNA (Figure 6). An interesting sensor for E. coli cell detection in drinking water has been developed based on the photodeposition of nanoparticulate silver on $\mathrm{a} \mathrm{TiO}_{2}$-coated 
piezoelectric quartz crystal electrode [57]. The silver coating enhances the binding of complementary DNA, allowing the sensor to detect a single $E$. coli cell in $100 \mathrm{~mL}$ of water.

A nanocomposite of $\mathrm{CeO}_{2}$ and $\mathrm{CH}$ has been used to immobilize an ssDNA probe for colorectal cancer genes using methylene blue as an indicator [58]. This NMO biocomposite shows excellent electronic conductivity and enhanced loading of the ssDNA probe. Sun et al. [59] have developed a novel and stable biosensor using $\mathrm{V}_{2} \mathrm{O}_{5}$ nanobelts, MWCNTs and a $\mathrm{CH}$ nanocomposite for the immobilization of ssDNA probes for the detection of Yersinia enterocolitica using DPV with methylene blue. This sensor can detect specific complementary DNA at concentrations of $0.01-1,000 \mathrm{nM}$ with a detection limit of $1.76 \mathrm{pM}$.

A DNA sensor based on a composite of MWCNTs, nanostructured $\mathrm{ZrO}_{2}$ and $\mathrm{CH}$ on GCE reported by Yang et al. [60] has high surface area and good charge-transport characteristics, providing increased DNA loading capacity and enhanced sensitivity. This DNA sensor shows a linear response to the logarithm of the target DNA concentration in the range of $0.149-93.2 \mathrm{nM}$ with a detection limit of $75 \mathrm{pM}$.

A nanocomposite of nanostructured shuttle-shaped $\mathrm{CeO}_{2}$, singlewalled nanotubes and hydrophobic room-temperature ionic liquid (1-butyl-3-methylimidazolium hexafluorophosphate) on a GCE has been used for electrochemical DNA hybridization detection [61]. The electron-transfer resistance of the electrode surface in this system increases after the immobilization of probe ssDNA. The biosensor has been used for the sequence-specific DNA detection of phosphoenolpyruvate carboxylase gene.

An electrochemical DNA biosensor based on a thin gold film sputtered onto anodic porous $\mathrm{NbO}_{x}$ has recently been reported [62]. This interesting biosensor shows enhanced biosensing performance with resolution 2.4 times higher than that of conventional bulk gold electrodes and a sensitivity three times higher. This sensor can be used for the detection of single-base mismatch DNA as well as for the discrimination of mismatch positions. Efforts should be made to improve the sensitivity of the nucleic acid sensor.

\section{Antibody-immobilized NMO-based immunosensors}

Electrochemical immunosensors have recently aroused much interest for the detection of proteins, biomarkers, biological toxins and biowarfare agents in critical situations such as food security, environmental assessment, pharmaceutical chemistry and clinical diagnostics. The immunosensors employ either antibodies or their complementary binding partners as biorecognition elements in combination with electrochemical transducers [63].

The surface-charged nanoporous morphology and efficient electrontransfer catalytic properties of NMOs make these materials useful as solid supports for antibody immobilization. In this architecture, the Fc terminal of an antibody binds with the NMO, and the Fab terminal is free to bind with the antigen with high specificity. To fabricate an NMObased immunosensor, the desired antibodies are immobilized onto an electronically conducting surface such as ITO. The sensing performance then depends on the density of antibodies immobilized on an appropriate surface. There is considerable scope for improving the functionality, electrical properties and morphology of biocompatible NMOs using novel chemical/physical routes for immunosensor fabrication [63-64].

A film of $\mathrm{IrO}_{2}$ grown electrochemically on a parent metal has been used as a substrate for an immunosensing electrode used in immunoassays for immunoglobulin $\mathrm{G}(\mathrm{IgG})[65]$. The $\mathrm{IrO}_{2}$ film in this case behaves as a hydrophilic and porous $3 \mathrm{D}$ matrix with high surface area. The stability of this immunosensor, however, has yet to be investigated. A pyrolytic graphite electrode bearing single-walled nanotubes and a self-assembled Nafion/ $/ \mathrm{Fe}_{2} \mathrm{O}_{3}$ coating has been used to immobilize anti-biotin antibodies to detect HRP-labeled and unlabeled biotin [66]. This sensor has a low detection limit due to the high surface area of SWCNT assemblies and the substrate's patternable conductivity. However, the sensing characteristics of this sensor have not been investigated. Hafaid et al. [67] reported an immunosensor based on magnetic functionalized $\mathrm{Fe}_{3} \mathrm{O}_{4}$ nanoparticles as a platform for biotin-streptavidin interaction. Surface plasmon resonance analysis has revealed a deviation of the measured angle during antigen-antibody recognition. Label-free detection based on EIS, on the other hand, allows variations in polarization resistance to be measured.

To investigate immunological interactions, Mantzila et al. fabricated anodic $\mathrm{Ti} / \mathrm{TiO}_{2}$ (anatase) electrodes at different potentials as an impedimetric measurement platform [68]. Wang et al. [69] fabricated an immunosensor based on hydrothermally prepared $\mathrm{TiO}_{2}$ nanowire bundles connected to a gold microelectrode by mask welding. Monoclonal antibodies immobilized on this platform provide sensitive, specific and rapid detection of Listeria monocytogenes at concentrations as low as $102 \mathrm{cfu} \mathrm{mL}^{-1}$ in just $1 \mathrm{~h}$ without significant interference from other food-borne pathogens. $\mathrm{TiO}_{2}$ arrays have also been utilized for label-free optical interferometric biosensing using proteins [70]. Immobilization in this case is achieved via electrostatic interactions between the negatively charged $\mathrm{TiO}_{2}$ surface and an IgG analyte for trinitrotoluene detection in the $\mathrm{pH}$ range $2-12$. Antibodies for prostate-specific antigens have been immobilized on modified $\mathrm{TiO}_{2}$ substrates [71]. The efficiency of this optical immunoassay-based biosensor is dependent on the silanization of the hydroxylated $\mathrm{TiO}_{2}$ substrate. The density of hydroxyl groups has been found to increase on exposure to oxygen plasma, resulting in high antibody loading and making this system suitable for label-free optical transduction.

A nanocomposite film of $\mathrm{TiO}_{2}$ and $\mathrm{CH}$ has been used for the preparation of an anti- $\alpha$-fetoprotein (AFP) sensor [72]. By immobilizing AFP, this sensor detects AFP in serum amperometrically with a linear range of $1.0-160 \mathrm{ng} \mathrm{mL}^{-1}$ and a low detection limit of $0.1 \mathrm{ng} \mathrm{mL}^{-1}$. An AFP sensor has also been prepared by immobilizing anti-AFP on the surface of gold nanoparticles electrodeposited on a $\mathrm{CH}-\mathrm{MnO}_{2} / \mathrm{MWNT}-\mathrm{Ag}$ composite on a GCE [73]. This immunosensor exhibits enhanced sensing characteristics, including a detection range of $0.25-250 \mathrm{ng} \mathrm{mL}^{-1}$ and detection limit of $0.08 \mathrm{ng} \mathrm{mL}^{-1}$, due to the high surface area and conductivity of MWCNT-Ag. The excellent film-forming ability of the $\mathrm{CH}-\mathrm{MnO}_{2}$ composite in this system prevents leakage and with gold nanoparticles provides a congenial microenvironment for anti-AFP.

A sol-gel-derived composite of a $\mathrm{CeO}_{2}$ film and nanostructured $\mathrm{ZnO}$ with a particle size of $-5.0 \mathrm{~nm}$ on ITO glass has been used for co-immobilization of rabbit $\operatorname{IgG}(\mathrm{r}-\mathrm{IgG})$ and bovine serum albumin (BSA) for ochratoxin A (OTA) detection by EIS [64]. This immunosensor exhibits linearity in the concentration range of $6-10 \mu \mathrm{M}$, a detection limit of $6 \mu \mathrm{M}$, a response time of $25 \mathrm{~s}$ and sensitivity of $189 \Omega \mathrm{nM}^{-1} \mathrm{~cm}^{-2}$ with a regression coefficient of 0.997 . A sol-gel derived nanostructured $\mathrm{CeO}_{2}$ film has also been used for the co-immobilization of $\mathrm{r}-\mathrm{IgG}$ and BSA to detect OTA [63]. A BSA/r-IgG/nano- $\mathrm{CeO}_{2} / \mathrm{ITO}$ immunoelectrode developed by Kaushik et al. [74] exhibits interesting characteristics such as a wide linear range $\left(0.5-6.0 \mathrm{ng} \mathrm{dL}^{-1}\right)$, low detection limit $\left(0.25 \mathrm{ng} \mathrm{dL}^{-1}\right)$, high sensitivity $\left(1.27 \mu \mathrm{Ang}^{-1} \mathrm{dL}^{-1} \mathrm{~cm}^{-2}\right)$ and a high association constant $\left(K_{\mathrm{a}}, 0.9 \times 10^{11} \mathrm{M}^{-1}\right)$, indicating the high affinity of the immunoelectrode for OTA. The improved sensitivity and detection limit of $\mathrm{CeO}_{2} / \mathrm{CH}$-based immunosensor have been attributed to the nanostructured surface and electrocatalytic properties of the NMO.

Kaushik et al. [75] also developed a nanocomposite in which fumed $\mathrm{SiO}_{2}$ nanoparticles form a $3 \mathrm{D}$ network in a $\mathrm{CH}$ matrix via hydrogen bonding. The availability of $\mathrm{NH}_{2} / \mathrm{OH}$ groups and the excellent filmforming ability of $\mathrm{CH}$ results in increased electron transport and high effective surface area, making this nanobiocomposite suitable for polyclonal $\mathrm{r}$-IgG immobilization. A nanobiocomposite platform of $\mathrm{r}$-IgG and BSA immobilized on $\mathrm{Fe}_{3} \mathrm{O}_{4}-\mathrm{CH}$ has been developed for OTA detection [76]. The charged surface $\mathrm{Fe}_{3} \mathrm{O}_{4}$ moieties provide a high electroactive surface area for loading of $\mathrm{IgG}$ and improved electron transport between $\mathrm{IgG}$ and the electrode. This $\mathrm{IgG} / \mathrm{CH}-\mathrm{Fe}_{3} \mathrm{O}_{4}$ nanobiocomposite on ITO immunoelectrode exhibits a fast response time and high sensitivity $\left(36 \mu \mathrm{Ang}^{-1} \mathrm{dL}^{-1} \mathrm{~cm}^{-2}\right)$.

$\mathrm{Au}-\mathrm{ZnO}$ nanocomposite films have been employed for enhanced surface plasmon resonance-based detection of tumor markers using 
carbohydrate antigen 15.3 [77]. The sensor responds to marker concentrations of $0.0125-160 \mathrm{U} \mathrm{mL}^{-1}$ in pleural fluid with high sensitivity, and the platform has been used to investigated bimolecular interactions.

$\mathrm{Fu}$ et al. [78] have developed a potentiometric immunoassay protocol for the determination of carcinoembryonic antigen (CEA) in human serum via immobilization of CEA monicolocal antibodies on $\mathrm{Fe}_{3} \mathrm{O}_{4}$ nanorods as a novel transparent immune affinity reactor. In this system, $\mathrm{Fe}_{3} \mathrm{O}_{4}$ 'bionanorods' with an average diameter of $40 \mathrm{~nm}$ and length of $1.0 \mu \mathrm{m}$ are used as the affinity support for the conjugation of anti-CEA antibodies. An electrochemical immunosensor based on dumbbell-like $\mathrm{Au}-\mathrm{Fe}_{3} \mathrm{O}_{4}$ nanoparticles has also been developed for the detection of the prostate-specific antigen (PSA) cancer biomarker [79]. This immunosensor has a sandwich-like structure that is prepared by immobilizing primary anti-PSA antibodies on a graphene surface and secondary anti-PSA antibodies on $\mathrm{Au}-\mathrm{Fe}_{3} \mathrm{O}_{4}$ nanoparticles. Signal amplification in this case is attributed to the effect of $\mathrm{Au}-\mathrm{Fe}_{3} \mathrm{O}_{4}$. There is considerable scope for improving $\mathrm{NMO}$-based immunosensors using monoclonal antibodies.

\section{Whole cell-immobilized NMO-based biosensors}

Gou et al. [80] investigated the mechanistic toxicity of nanostructured silver and $\mathrm{TiO}_{2}$ anatase using a prokaryotic real-time gene expression profiling method. These NMOs were chosen for the study because of their widespread use in the detection of analytes, and were examined in experiments relating to the properties of compound-specific and concentration-sensitive two-dimensional (genes and time) gene expression using nanostructured $\mathrm{TiO}_{2}$.

\section{Conclusions and future prospects}

Nanostructured metal oxide-based biosensors provide a new horizon for novel functions with a variety of applications in clinical and non-clinical diagnostics. Various NMO particles have already made a major impact in various applications, ranging from enzyme electrodes to genoelectronics. The useful properties of NMOs suggest that future interdisciplinary research is likely to lead to a new generation of electrochemical biosensors. Researchers are now focusing on understanding the various biomolecule-transducer interactions using these interesting nanomaterials. Embedded particle carriers, nanoparticle-based DNA barcodes, nanoparticle-based surface plasmon resonance and chemiluminescence detection, and biocatalytic metallization for amplified assay studies should all be regarded as promising research targets for the development of amplified bioaffinity assays. Highly sensitive biodetection protocols based on NMOs open up the possibility of creating biosensors for disease markers, biological and infectious agents in early-stage detection of disease and threats. DNA-, enzyme- and antibody-based bioassays on nanoparticulate MO platforms are also expected to be useful for multianalyte detection. In this context, it will be interesting to explore the dependence of size and toxicity of MO nanoparticles in a variety of new applications.

A wide range of new NMOs is expected to further expand the area of biosensors. New strategies for the synthesis of one-dimensional NMOs are likely to result in new bioelectronic sensing applications. The ability to functionalize NMOs with the desired groups to bind target biomolecules, and doping these with electronically active materials to obtain enhanced charge transfer, may pave the way for new methods for biosensor development. The synthesis of MO nanoparticles with various morphologies at the nanodomain level may provide a suitable environment for obtaining the oriented immobilization of desired biomolecules and thereby amplified signals. These NMOs can be fabricated and tested in desired patterns, such as sensor arrays, for the development of functional integrated devices. The interface of NMObased devices could also be used for parallel real-time monitoring of multiple analytes. It will be interesting to focus on new methods for the fabrication of innovative biosensor arrays with desired properties for health care, confining different biomolecules onto closely spaced one-dimensional nanostructures based on NMOs.

\section{Acknowledgments}

The authors thank R. C. Budhani of the National Physical Laboratory, New Delhi, India, for providing facilities. Pratima R. Solanki and Ajeet Kaushik gratefully acknowledge the Department of Science and Technology (DST), India, for financial support. This review was prepared with the financial support of the DST (DST/TSG/ ME/2008/18 and GAP-070932) and the Department of Biotechnology of the Government of India (DBT/GAP070832).

\section{References}

1. F. Caruso, Adv. Mater. 13, 11 (2001)

2. P. Pandey, M. Datta, B. D. Malhotra, Anal. Lett. 41, 159 (2008).

3. C. Jianrong, M. Yuqing, H. Nongyue, W. Xiaohua, L. Sijiao, Biotechnol. Adv 22, 505 (2004)

4. S. P. Singh et al., Appl. Phys. Lett. 91, 063901 (2007).

5. A. K. Gupta, M. Gupta, Biomaterials 26, 3995 (2005).

6. A. A. Ansari, P. R. Solanki, B. D. Malhotra, Appl. Phys. Lett. 92, 263901 (2008).

7. J. Liu, Y. Li, X. Huang, Z. Zhu, Nanoscale Res. Lett. 5, 1177 (2010).

8. H. J. Kim, S. H. Yoon, H. N. Choi, Y. K. Lyu, W. Y. Lee, Bull. Korean Chem. Soc. 27, 65 (2006).

9. R. Doong, H. Shih, Biosens. Bioelectron. 25, 1439 (2010).

10. R. Tenne, Nat. Nanotechnol. 1, 103 (2006).

11. H. N. Choi, M. A. Kim, W. Y. Lee, Anal. Chim. Acta 537, 179 (2005).

12. Z. Zhao, W. Lei, X. Zhang, B. Wang, H. Jiang, Ah. S. Sens. 10, 1216 (2010).

13. J. Wang, Small 1, 1036 (2005).

14. J. Wang, Analyst 130, 421 (2005).

15. S. E. McNeil, J. Leukocyte Bio. 78, 585 (2005).

16. C. Pan, J. Zhu, J. Mater. Chem. 19, 869 (2009).

17. M. M. Rahman, A. J. S. Ahammad, J. H. Jin, S. J. Ahn, J. J. Lee, Sensors 10, 4855 (2010).

18. A. Chaubey, B. D. Malhotra, Biosens. Bioelectron. 17, 441 (2002).

19. A. E. Nel et al., Nat. Mater. 8, 543 (2009).

20. J. X. Wang et al., Appl. Phy. Lett. 88, 233106 (2006).

21. T. Kong et al., Sensor. Actuator. B 138, 344 (2009).

22. M. Ahmed, C. Pan, Z. Luo, J. Zhu. J. Phys. Chem. C 114, 9308 (2010).

23. J. Liu, Y. Li, X. Huang, Z. Zhu, Nanoscale Res. Lett. 5, 1177 (2010).

24. M. H. Asif et al., Biosens. Bioelectron. 25, 2205 (2010).

25. S. Saha et al., Biosens. Bioelectron 24, 2040 (2009).

26. D. Wen, S. Guo, Y. Wang, S. Dong, Langmuir 26, 11401 (2010).

27. D. Wan et al., J. Phys. Chem. C 113, 13023 (2009).

28. A. Kaushik et al., Biosens. Bioelectron. 24, 676 (2008).

29. A. Umar, M. M. Rahman, Y. B. Hahn, Electrochem. Commun. 11, 1353 (2009).

30. A. Salimi, E. Sharifi, A. Noorbakhsh, S. Soltanian, Biosens. Bioelectron. 22, 3146 (2007).

31. P. R. Solanki, A. Kaushik, A. A. Ansari, B. D. Malhotra, Appl. Phys. Lett. 94, 143901 (2009).

32. M. Ahmad, C. Pan, L. Gan, Z. Nawaz, J. Zhu, J. Phys. Chem. C 114, 243 (2010).

33. A. Umar, M. M. Rahman, M. Vaseem, Y.-B. Hahn, Electrochem. Communi. 11, 118 (2009)

34. A. A. Ansari, A. Kaushik, P. R. Solanki, B. D. Malhotra, Electroanal. 21, 965 (2009).

35. A. Salimi, R. Hallaj, S. Soltanian, Electroanal. 21, 2693 (2009).

36. A. Kaushik et al., Electroanal. 22, 1045 (2010).

37. C. Xia, N. Wang, L. Lidong, G. Lin, Sensor. Actuat. B-Chem 129, 268 (2008).

38. C. Xiang, Y. Zou, L.-X. Sun, F. Xu, Sensor. Actuat. B-Chem 136, 158 (2009).

39. B. X. Gu et al., J. Phys. Chem. B113, 6553 (2009).

40. A. A. Ansari, P. R. Solanki, B. D. Malhotra, J. Biotechnol. 142, 179 (2009).

41. L. Lu et al., Electroanal. 22, 471 (2010).

42. J. Liu et al., Electrochem. Commun.11, 202 (2009).

43. A. Ali et al., Mater. Lett. 63, 2473 (2009).

44. P. R. Solanki, A. Kaushik, A. A. Ansari, G. Sumana, B. D. Malhotra, Appl. Phys. Lett. 93, 163903 (2008)

45. A. Kaushik et al., Sensor. Actuat. B-Chem 138, 572 (2009).

46. A. Vijayalakshmi et al., Biosens. Bioelectron. 23, 1708 (2008).

47. P. R. Solanki et al., Sensor. Actuat. B-Chem 141, 551 (2009).

48. Y.-H. Bai, H. Zhang, J.-J. Xu, H.-Y. Chen, J. Phys. Chem. C 112, 18984 (2008).

49. Y. Luo, H. Liu, Q. Rui, Y. Tian, Anal. Chem. 81, 3035 (2009).

50. J. Wang, S. Li, Y. Zhang, Electrochim. Acta 55, 4436 (2010).

51. W. Zhang, T. Yang, D. M. Huang, K. Jiao, Chinese Chem. Lett. 19, 589 (2008).

52. H. Zhu, J. Wang, G. Xu, Cryst. Growth. Des. 9, 633 (2009). 
53. S.-H. Zuo et al., Bioelectrochemistry 74, 223 (2009).

54. M. Das, G. Sumana, R. Nagarajan, B. D. Malhotra, Appl. Phys. Lett. 96, 133703 (2009)

55. N. Prabhakar, P. R. Solanki, A. Kaushik, M. K. Pandey, B. D. Malhotra, Electroanal. 22, 2672 (2010).

56. P. R. Solanki, A. Kaushik, P. M. Chavhan, S. N. Maheshwari, B. D. Malhotra, Electrochem. Commun. 11, 2272 (2009).

57. H. Sun, T. S. Choy, D. R. Zhu, W. C. Yam, Y. S. Fung, Biosens. Bioelectron. 24, 1405 (2009).

58. K.-J. Feng et al., Talanta 70, 561 (2006).

59. W. Sun, P. Qin, H. Gao, G. Li, K. Jiao, Biosens. Bioelectron 25, 1264 (2010).

60. Y. Yang et al., Anal. Chim. Acta 584, 268 (2007).

61. W. Zhang, T. Yang, X. Zhuang, Z. Guo, K. Jiao, Biosens.Bioelectron 24, 2417 (2009)

62. S. Rho, et al., Biosens. Bioelectron. 23, 852 (2008)

63. A. Kaushik, P. R. Solanki, A. A. Ansari, S. Ahmad, B. D. Malhotra, Nanotechnol. 20, 055105 (2009)

64. A. A. Ansari, A. Kaushik, P. R. Solanki, B. D. Malhotra, Bioelectrochemistry 77,75 (2010).
65. M. S. Wilson, R. D. Rauh, Biosen. Bioelectron 19, 693 (2004).

66. M. O'Connor et al., Analyst 129, 1176 (2004).

67. I. Hafaid et al., J. Sensors 2009, 746548 (2009).

68. A. G. Mantzila , M. I. Prodromidis, Electrochim. Acta 51, 3537 (2006).

69. R. Wang, C. Ruan, D. Kanayeva, K. Lassiter, Y. Li, Nano Lett. 8, 2625, (2008).

70. K.-S. Mun, S. D. Alvarez, W.-Y. Choi, M. J. Sailor, ACS Nano 4, 2070 (2010).

71. Y. Zhang et al., J. Mater. Chem. 19, 948 (2009).

72. L. Tan et al., Sensor. Actuator. B-Chem. 142, 316 (2009).

73. X. Che et al., J. Colloid Interf. Sci. 345, 174 (2010).

74. A. Kaushik, P. R. Solanki, M. K. Pandey, S. Ahmad, B. D. Malhotra, Appl. Phys. Lett. 95, 173703 (2009)

75. A. Kaushik, P. R. Solanki, K. N. Sood, S. Ahmad, B. D. Malhotra, Electrochem. Commun. 11,1919 (2009)

76. A. Kaushik, P. R. Solanki, A. A. Ansari, S. Ahmad, B. D. Malhotra, Electrochem Commun. 10, 1364 (2008).

77. C.-C. Chang et al., Anal. Chem. 82, 1207 (2010).

78. X. Fu, J. Wang, N. Li, L. Wang, L. Pu, Microchim. Acta 165, 437 (2009).

79. Q. Wei et al., Biosens. Bioelectron. 26, 627 (2010).

80. N. Gou, A. O. Haydeng, A. Z. Gu, Envir. Sci. Technol. 44, 5964 (2010).

\section{Author profiles}

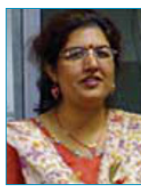

\section{Pratima R. Solanki}

Pratima R. Solanki obtained her MSc and PhD degrees in bioscience from Maharishi Dayanand University, Rohtak, India, in 1995 and 2000. She is working as a Department of Science and Technology Young Scientist with the Biomedical Instrumentation Section of the National Physical Laboratory, Council of Scientific Industrial Research, New Delhi, India. She is actively engaged in the research and development of biosensors for healthcare and environmental monitoring.

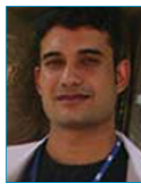

\section{Ajeet Kaushik}

Ajeet Kaushik has submitted his doctoral thesis in the area of organic-inorganic hybrid nanocomposites for sensing application at Jamia Millia Islamia, New Delhi, India. He is working as research fellow in the Biomedical Instrumentation Section of the National Physical Laboratory, Council of Scientific Industrial Research, New Delhi, India. He is actively engaged in the research and development of biosensors for healthcare.

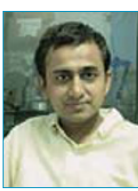

\section{Ved V. Agrawal}

Ved V. Agrawal earned his MSc degree at the Indian Institute of Technology Delhi in 2002 and his PhD degree from the Jawaharlal Nehru Center for Advanced Scientific Research in 2008, where he investigated ultrathin nanocrystalline films generated at liquid-liquid interfaces. In 2008, he began postdoctoral studies in the Thin Film lab at the Indian Institute of Technology Delhi. He now works at the National Physical Laboratory, Council of Scientific Industrial Research, New Delhi, India. His current research focuses on utilizing the properties of nanomaterials to improve biosensing characteristics.

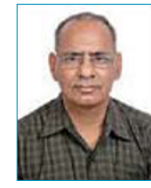

\section{Bansi D. Malhotra}

Bansi D. Malhotra received his PhD degree in physics from University of Delhi, Delhi, India in 1980. He is currently head of the Department of Science and Technology Centre on Biomolecular Electronics at the National Physical Laboratory, Council of Scientific Industrial Research, New Delhi, India. He holds ten patents in the area of biomolecular electronics. His current activities include research on biosensors, conducting polymers, Langmuir-Blodgett films, self-assembled monolayers, nanomaterials and bimolecular electronics. 